\title{
ACID-STABLE SERINE PROTEINASE INHIBITORS IN THE URINE OF ALZHEIMER DISEASE SUBJECTS
}

\author{
GIULIA SPARRO, SALVATORE BONAIUTO*, GABRIELLA GALDENZI, \\ ANNA MARIA ELEUTERI, MAURO ANGELETTI, GIULIO LUPIDI, ROSALIA TACCONI, \\ ELVIO GIANNANDREA ${ }^{\circ}$, ANDREA VESPRINI*, EVANDRO FIORETTI \\ Department of Molecular, Cellular and Animal Biology, Post-Graduate School in Biochemistry and \\ Clinical Chemistry, University of Camerino, 62032-Camerino (MC), Italy. \\ * Italian National Research Centers on Aging (INRCA) of Fermo*, 63023 Fermo (AP) and Appignano ${ }^{\circ}$, \\ 62010 Appignano (MC), Italy.
}

\section{SUMMARY}

\begin{abstract}
A comparative study of the levels of acid-stable proteinase inhibitors (kallikrein and trypsin inhibitors) in the urine of healthy and Alzheimer subjects, of both sexes, has been performed. A preliminary characterization of the purified inhibitors indicates that the urinary antitryptic activity is accounted for by the presence of the well known Urinary Trypsin Inhibitor (UTI) while an apparently new molecule appears to be responsible for the antikallikrein activity. The urinary levels of kallikrein inhibitors are very similar in healthy and sick subjects while the levels of trypsin inhibitors appear significatively increased in Alzheimer subjects of both sexes. The data presented here support the hypothesis that unpaired proteolytic processes could be involved in the pathogenesis of Alzheimer's disease and suggest that the levels of urinary acid-stable inhibitors may prove to be useful markers of the disease.
\end{abstract}

KEY WORDS Proteinase inhibitors Alzheimer disease Proteolysis

\section{INTRODUCTION}

Imbalance in the dynamic equilibrium between active proteinases and their specific inhibitors is suspected to be a pathogenic factor for many human diseases (Laskowski and Kato, 1980; Fritz and Wunderer, 1983; Travis and Salvesen, 1983; Jochum et al., 1986).

A particularly significative association appears to be that between some malignancies and the expression of low molecular weight trypsin inhibitors such as the Pancreatic Secretory Trypsin Inhibitor (PSTI) (Matsuda et al., 1983; Huhtala et al., 1982; Ogawa et al., 1987; Tomita et al., 1990) and the so called Acid Stable Trypsin Inhibitor (ASTI) Okumichi et al., 1984; Onitsuka et al., 1985; Sumi et al., 1987; Yoshida et al., 1989). The latter, a multipotent inhibitor found in several biological fluids, including urine, corresponds to the Urinary Trypsin Inhibitor (UTI) (Shulman, 1955; Gebhard and Hochstrasser, 1986) and can be obtained as a product of acid, or enzymatic, hydrolysis from the large plasma protein Inter- $\alpha$-Trypsin Inhibitor (ITI) (Gebhard and Hochstrasser, 1986). Owing to its antifibrinolytic activity, UTI appears to play an important role in the metabolism of tumour cells and levels are increased in the urine of cancer patients (Chawla et al., 1978; Onitsuka et al., 1985).

Correspondence to: Prof. Evandro Fioretti, Department of Molecular, Cellular and Animal Biology, Univ. of Camerino, 62032 CAMERINO (MC), ITALY. Tel.: +39-737-40715; fax: +39-737-636822. 
Recently a role has been proposed for abnormalities of proteolysis in the aetiology of Alzheimer's disease, a degenerative condition, mainly characterized by the presence of neuritic plaques in several brain regions (Abraham and Potter, 1989). It has been suggested that these proteinaceous deposits could originate from an aberration of the proteolysis in the brain (Masters et al., 1985). Supportive evidence has been deduced from the finding, in the neuritic plaque, of the serine proteinase inhibitor $\alpha_{1}$-antichymotrypsin (Abraham et al., 1988) and a 57 residue domain resembling the kallikrein inhibitor, aprotinin or "Kunitz inhibitor" (Fritz and Wunderer, 1983) within the precursor of the $\beta$-amyloid protein (Ponte et al., 1988, Tanzi et al., 1988; Kitaguchi et al., 1988), the most abundant protein component of the plaque. Recently, it has been reported that, in proteolytic processing of the $\beta$-amyloid precursor, a proteinase very similar to cathepsin $\mathrm{G}$ could be involved (Razzaboni et al., 1992). This enzyme which is expressed in astrocytes (Razzaboni et al., 1992) in intimate contact with the neuritic plaque, could be regulated by UTI-like inhibitors (Gebhard and Hochstrasser, 1986) and kallikrein inhibitors (Razzaboni et al., 1992).

We have recently found that acid treated human urine contains antiproteolytic activities against kallikrein (Eleuteri et al., 1994), a serine proteinase which appears to play an important role also in cerebral function (Kizuki et al., 1994). We have, therefore, undertaken a comparative study of the levels of acid-stable proteinase inhibitors (kallikrein and trypsin inhibitors) in the urine of healthy and Alzheimer subjects.

\section{MATERIALS AND METHODS}

\section{Chemicals}

Bovine trypsin, and the synthetic substrate N- $\alpha$-Benzoyl-DL-Arginine-p-Nitroanilide (BzArgNan) were obtained from Sigma Chemical Co (USA). Porcine pancreas kallikrein and aprotinin were a kind gift of Bayer AG (Germany). The synthetic substrate S-2266 used for measuring the kallikrein activity was obtained from Kabi Vitrum (Sweden). Antibodies anti-human Inter- $\alpha$-Trypsin Inhibitor were obtained from Dakopatts (Denmark) and utilized without further purification. Polyclonal antibodies against aprotinin, raised in rabbits, were kindly supplied by Bayer AG (Germany). Sep-Pak $\mathrm{C}_{18}$ cartridges were obtained from Millipore (USA), and prepacked HiTrap ${ }^{\mathrm{TM}}$ affinity columns (1 ml volume) from Pharmacia (Sweden). All other chemicals were of analytical grade.

\section{Urine samples}

Urine samples, collected in the morning, were obtained from healthy or Alzheimer subjects of both sexes (five males and five females) of comparable age, and were treated as soon as possible after collection. Quantitation of the inhibitors was performed in triplicate on three different samples for each subject considered. Dementia was diagnosed according to the criteria of the Diagnostical Statistical Manual of mental disorders (DSM-III-Revised) (American Psychiatric Association, 1980). The subjects were classified as affected by Alzheimer's disease when the criteria reported by the NINCDSADRDA work group were satisfied (McKhann et al., 1984).

\section{Affinity chromatography}

Porcine pancreas kallikrein, or bovine trypsin were immobilised on HiTrap ${ }^{\mathrm{TM}}-\mathrm{NHS}$ activated columns according to the suppliers' instructions. 


\section{Isolation of the inhibitors}

Isolation of the acid-stable urinary inhibitors was carried out taking advantage of a procedure which allows the quantitative recovery of small amounts of active inhibitors in biological fluids essentially based on affinity- and reversed phase-HPLC chromatography as major purification tools (Fioretti et al., 1993) (see Scheme 1).

\section{HPLC}

The HPLC analyses were performed with a System Gold apparatus (Beckman, USA) using a reversed phase (RP) column Supelcosil LC 18-DB, equipped with a guard column, both obtained from Supelco (USA). The column was equilibrated in $0.1 \%$ trifluoroacetic acid (TFA) in water (solvent A) and eluted with a gradient obtained by mixing solvent A with a solution of $4: 1(\mathrm{v} / \mathrm{v})$ acetonitrile-2-propanol in $0.1 \%$ TFA (solvent B) at a flow-rate of $1 \mathrm{ml} / \mathrm{min}$. The inhibitor samples were prepared by dissolution with solvent A just before use. Absorbance of the eluted fractions was recorded at 254 $\mathrm{nm}$. Chromatographic data were analyzed with the Gold software.

\section{Concentration of the inhibitors}

The concentration of the urinary inhibitors was determined by titration with an aprotinin standardized trypsin solution, as previously reported (Fioretti et al., 1993).

\section{Antiproteolytic activity}

The antiproteolytic activity of the urinary inhibitors, in the HPLC eluates, was determined by measuring their antitrypic or/and antikallikrein activity as previously reported (Fioretti et al., 1993). The presence of organic solvents, which affects the proteolytic activity, was taken into account adding, in the reference test, an eluate volume from a run performed without the inhibitor.

\section{Immunochemical characterization of the inhibitors}

Cross-reactivity between polyclonal antibodies raised against bovine aprotinin, or human ITI, and the urinary inhibitors was assayed as previously reported (Fioretti et al., 1984; Fioretti et al., 1985) measuring the ability of the antibodies to remove the antitryptic activity of the inhibitors.

\section{RESULTS}

The experimental procedure utilized for the isolation of the acid-stable urinary proteinase inhibitors is summarized in Scheme 1. Usually, 200-250 ml of freshly collected urine was acidified by slow addition, under stirring, of a concentrated solution (50\%) of trichloroacetic acid to a final TCA concentration of $2.5 \%$. The supernatant obtained after centrifugation, was brought to $\mathrm{pH} 8.6$ with concentrated $\mathrm{NaOH}$ solution and applied to a $1 \mathrm{ml}$ prepacked HiTrap ${ }^{\mathrm{TM}}-\mathrm{kallikrein}$ column, for isolation of the kallikrein inhibitors, or, alternatively, to a $1 \mathrm{ml} \mathrm{HiTrap}{ }^{\mathrm{TM}}$-trypsin column for the isolation of the trypsin inhibitors. Both columns were washed with the equilibrium buffer $(200 \mathrm{mM}$ Tris- $\mathrm{HCl} \mathrm{pH} 8.6$ containing $20 \mathrm{mM} \mathrm{CaCl}_{2}$ ) and eluted with a solution of $\mathrm{HCl}$ at $\mathrm{pH} 2.0$. In both cases, the fractions showing antiproteolytic activity (measured as antitryptic activity) were pooled, neutralized and loaded on a Sep-Pak $\mathrm{C}_{18}$ cartridge. The cartridge was eluted with a mixture obtained by mixing $60 \%$ of Solvent A and $40 \%$ of Solvent B 
Scheme 1. Procedure adopted for the isolation of the urinary acid-stable proteinase inhibitors.

Freshly collected urine

Trichloroacetic acid precipitation

Kallikrein affinity

chromatography

bound material

RP-Chromatography

(Sep-Pak $\mathrm{C}_{18}+\mathrm{C}_{18}$ HPLC column)

Kallikrein inhibitors
Trypsin affinity chromatography

bound material

RP-Chromatography

(Sep-Pak $\mathrm{C}_{18}+\mathrm{C}_{18}$ HPLC column)

Trypsin inhibitors

(see Materials and Methods). This fraction (about $3.0 \mathrm{ml}$ ) was utilized for the quantitative determination of the inhibitors in the urine samples (see Table 1). The determination was carried out using a titration with an aprotinin-standardized trypsin solution (see Material and Methods) taking advantage of the high affinity $\left(\mathrm{Kd}<10^{-11} \mathrm{M}\right)$ shown by both kinds of urinary inhibitors for the enzyme (see also below). Further information on the nature of the inhibitors recovered from the affinity columns was obtained through an HPLCreversed phase (RP) analysis and a preliminary molecular characterisation. The fraction obtained from the Sep-Pak $\mathrm{C}_{18}$ cartridge was concentrated to dryness, dissolved in $50 \mu \mathrm{l}$ of Solvent $\mathrm{A}$ and then loaded on a RP- $\mathrm{C}_{18}$ column, equilibrated and eluted as reported in the Material and Methods section. Figure 1 reports the elution profile and the antiproteolytic activity, determined against kallikrein and trypsin, of the fractions eluted from the $\mathrm{C}_{18}$ column when the material recovered from the kallikrein affinity column was chromatographed. In this case, the fractions showing antikallikrein activity (hereafter termed "kallikrein inhibitors") are eluted from the column at a concentration ranging between $30 \%$ and $40 \%$ of Solvent B, and both the elution profile and the antikallikrein activity appear very similar when the urine of Alzheimer (Figure 1B) or control subjects (Figure 1A) was analyzed. The kallikrein inhibitors also show antitryptic activity and their antiproteolytic activity is unaffected either by the presence of polyclonal antibodies raised against the bovine aprotinin or by anti-human ITI antibodies which are immunologically identical to anti-UTI antibodies (Gebhard and Hochstrasser, 1986) (see above).

These results prompted further investigation of the nature of urinary kallikrein inhibitors. The fraction recovered from the $\mathrm{C}_{18}$ column was subjected either to SDSpolyacrylamide gel electrophoresis in non reducing conditions (Laemmli, 1970) or to gel permeation chromatography on a Superdex 75 HR 10/30 column (Pharmacia, Sweden). 
Table 1. Urine content of acid-stable, low molecular weight, proteinase inhibitors in Alzheimer and healthy subjects.

\begin{tabular}{lcccr}
\hline & \multicolumn{2}{c}{ Alzheimer } & \multicolumn{2}{c}{ Control } \\
& Male & Female & Male & Female \\
\hline Age (years) & $80-90$ & 80 & $80-90$ & 80 \\
Kallikrein inhibitors $(\mu \mathrm{g} / \mu \mathrm{l})$ & $10 \pm 1$ & $8 \pm 1$ & & $10 \pm 1$ \\
& & & $9 \pm 1$ & \\
Trypsin inhibitors $(\mu \mathrm{g} / \mu \mathrm{l})$ & $480 \pm 5$ & $230 \pm 5$ & $143 \pm 4$ & $130 \pm 5$ \\
\hline
\end{tabular}

Inhibitor content was determined on the basis of antitryptic residual activity using an aprotinin standardized trypsin solution. Determinations were performed in triplicate on the fractions recovered from the Sep-Pak $\mathrm{C}_{18}$ cartridge (see Materials and Methods).

Both analyses showed that this fraction contained several proteins and that the antikallikrein activity was associated to a protein showing an apparent molecular weight of about $24 \mathrm{KDa}$. The complete characterization of this inhibitor is in the progress.

When the material recovered from the immobilized trypsin column was chromatographed on the RP-C ${ }_{18}$ HPLC column under the conditions reported, the antiproteolytic activity was found in fractions eluted at a concentration ranging between $30 \%$ and $80 \%$ of Solvent B (Figure 2). As in the previous case, these fractions were assayed for anti-kallikrein activity and for their cross reactivity towards anti-aprotinin and anti-ITI antibodies. The results obtained clearly indicate that, in this case, two kinds of trypsin inhibitors are present in the $\mathrm{C}_{18}$ elute: i) a first one eluted between $30 \%$ and $40 \%$ of solvent $\mathrm{B}$, accounting for the presence of the kallikrein inhibitors (see above) which shows also antitryptic activity and a second one (hereafter termed the "trypsin inhibitors") eluted between $40 \%$ and $80 \%$ of solvent $\mathrm{B}$, which is inactive against kallikrein and recognizes anti-human-ITI antibodies (Figure 3). Also in this case, both the elution profile and the antiproteolytic activity of the fractions eluted when urine of Alzheimer (Figure 2B) or control subjects (Figure 2A) was subjected to HPLC analysis, appear very similar. The urinary antitryptic activity is associated, as expected, with multiple protein peaks showing an apparent molecular weight of $30 \mathrm{KDa}, 24 \mathrm{KDa}$ and $18 \mathrm{KDa}$ respectively, most likely arising from a proteolytic fragmentation of the native Inter- $\alpha$-Trypsin Inhibitor. As can be seen in Table 1, where the results obtained from this study are summarized, striking differences in the urinary levels of the kallikrein and trypsin inhibitors were observed. The urine content of kallikrein inhibitors (about $10 \mu \mathrm{g} / \mathrm{l}$ ) was very similar for healthy and Alzheimer subjects of both sexes, while in ill subjects a marked increase in the levels of the trypsin inhibitors was observed. As can be seen in Table 1 a mean content of $135 \mu \mathrm{g}$ of trypsin inhibitors per litre of urine was found in control subjects $(150 \mu \mathrm{g} / \mathrm{litre}$ for males and $120 \mu \mathrm{g} /$ litre for females). This value is elevated 2-3 fold in the urine of Alzheimer patients 

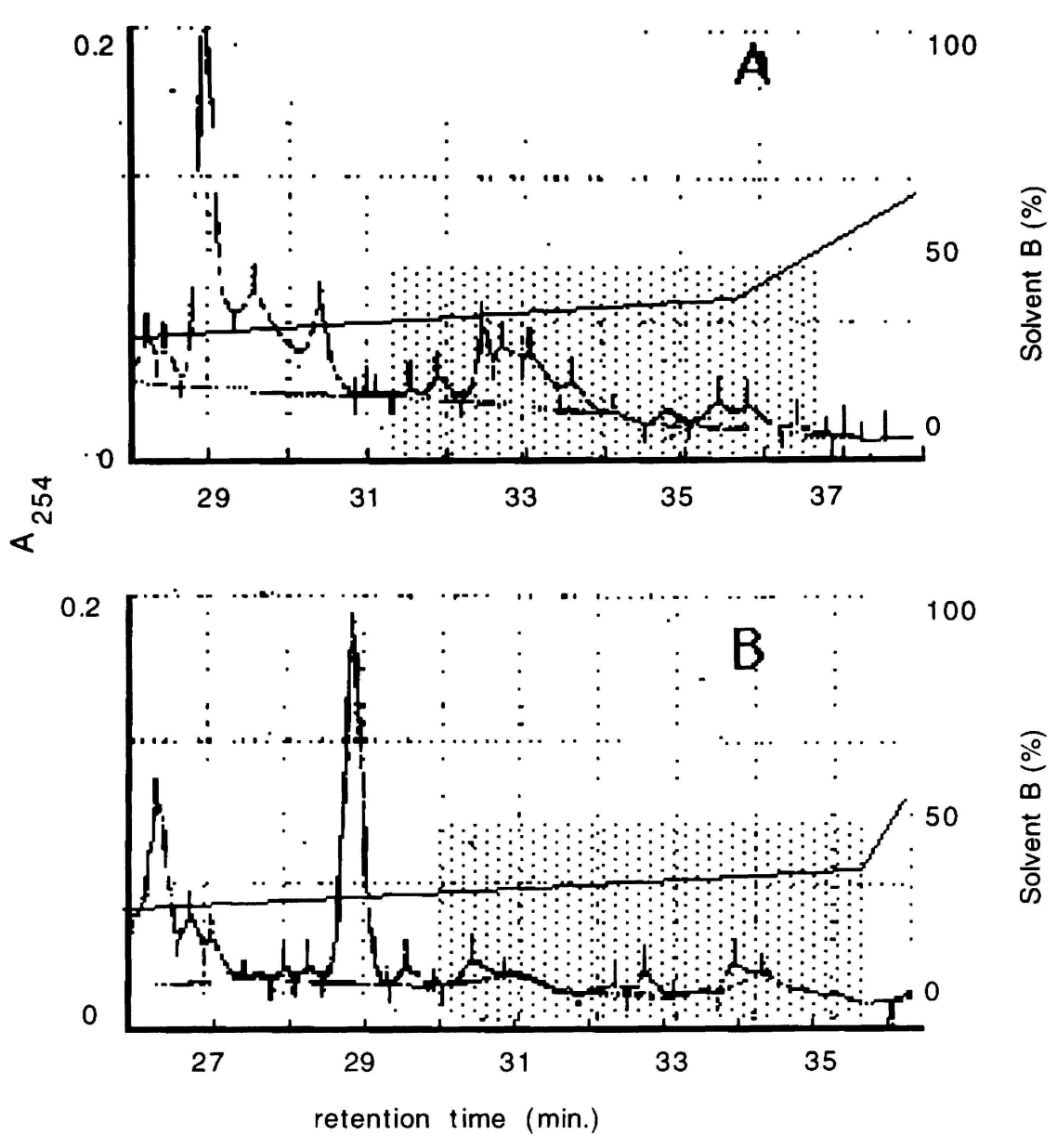

Figure 1. HPLC elution profile and antiproteolytic activity of the urinary kallikrein inhibitors isolated from the urine of Alzheimer (B) or control subjects (A). Column: Supelcosil LC ${ }_{18}$-DB. Flow-rate: $1.0 \mathrm{ml} / \mathrm{min}$. Detection wavelength: $254 \mathrm{~nm}$. The shaded area accounts for the fractions showing antiproteolytic activity determined against kallikrein and trypsin. For other details see Materials and Methods.

\section{DISCUSSION}

A correct balance between proteolytic and antiproteolytic activities appears important for many physiological processes, those occurring at brain level included. Proteinases and proteinase inhibitors have, in fact, been involved in several aspects of neurogenesis and in glial function (Pittman, 1984; Krystosek and Seeds, 1984; Pittman, 1985; Pittman, 1987). A more direct involvement has recently been reported for the serine proteinases kallikrein (Kizuki et al., 1994) and cathepsin G (Razzaboni et al., 1992). Immunoreactivity for glandular kallikrein was found in both the cerebral cortex and the brain stem of rats and appeared to be concentrated in the neuronal cell bodies, indicating, for this protease, an involvement in neuron function (Kizuki et al., 1994). In addition, a marked decrease in kallikrein activity has been observed in the brain of Alzheimer subjects 

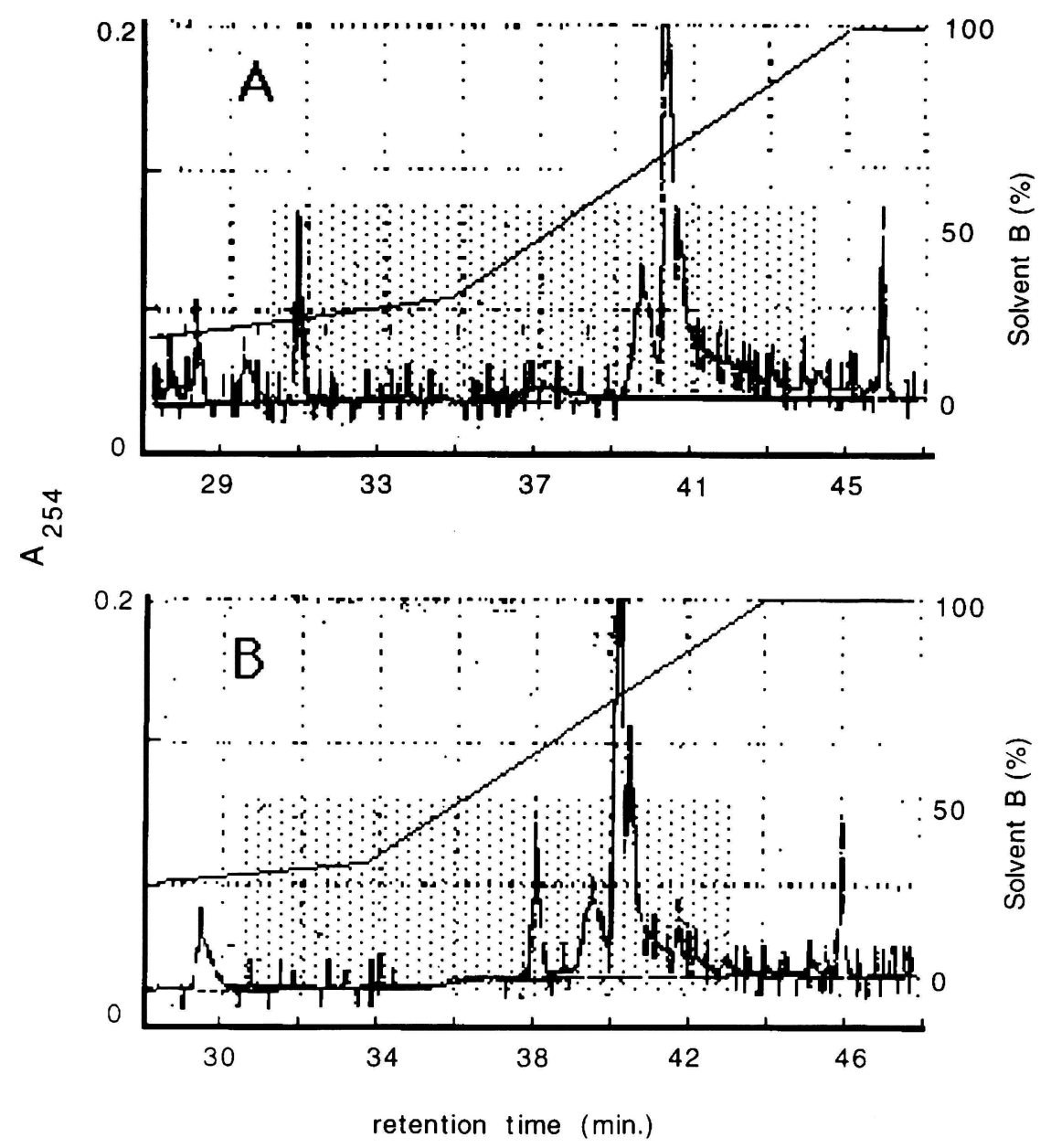

Figure 2. HPLC elution profile and antiproteolytic activity of the urinary trypsin inhibitors isolated from the urine of Alzheimer (B) or control subjects (A). Column: Supelcosil LC $_{18}$-DB. Flow-rate: $1.0 \mathrm{ml} / \mathrm{min}$. Detection wavelength: $254 \mathrm{~nm}$. The shaded area accounts for the fractions showing antitryptic activity. For other details see Materials and Methods.

(Aoyagy et al.. 1990) as well as in old senescence-accelerated mice (Kizuki et al., 1994). A cathepsin G-like activity has been partially purified from the normal human and monkey brain and from the brain of Alzheimer subjects (Razzaboni et al., 1992); this proteolytic activity appears to be sensitive to inhibition by the kallikrein inhibitor (aprotinin) and is localized in astrocytes, glial cells surrounding the neuritic plaque (Razzaboni et al., 1992). Owing to this localization it has been hypothesized that this proteinase could be involved in processing the $\beta$-amyloid precursor and hence could influence formation and deposition of the $\beta$-amyloid. We now show that, in human urine, acid-stable proteinase inhibitors showing antikallikrein or antitrypsin activity are present and that their levels, at least for the trypsin inhibitors, are increased in subjects with Alzheimer's disease. Both kallikrein-and trypsin inhibitors show a broad specificity towards proteolytic enzymes and are active against proteinases apparently involved in 


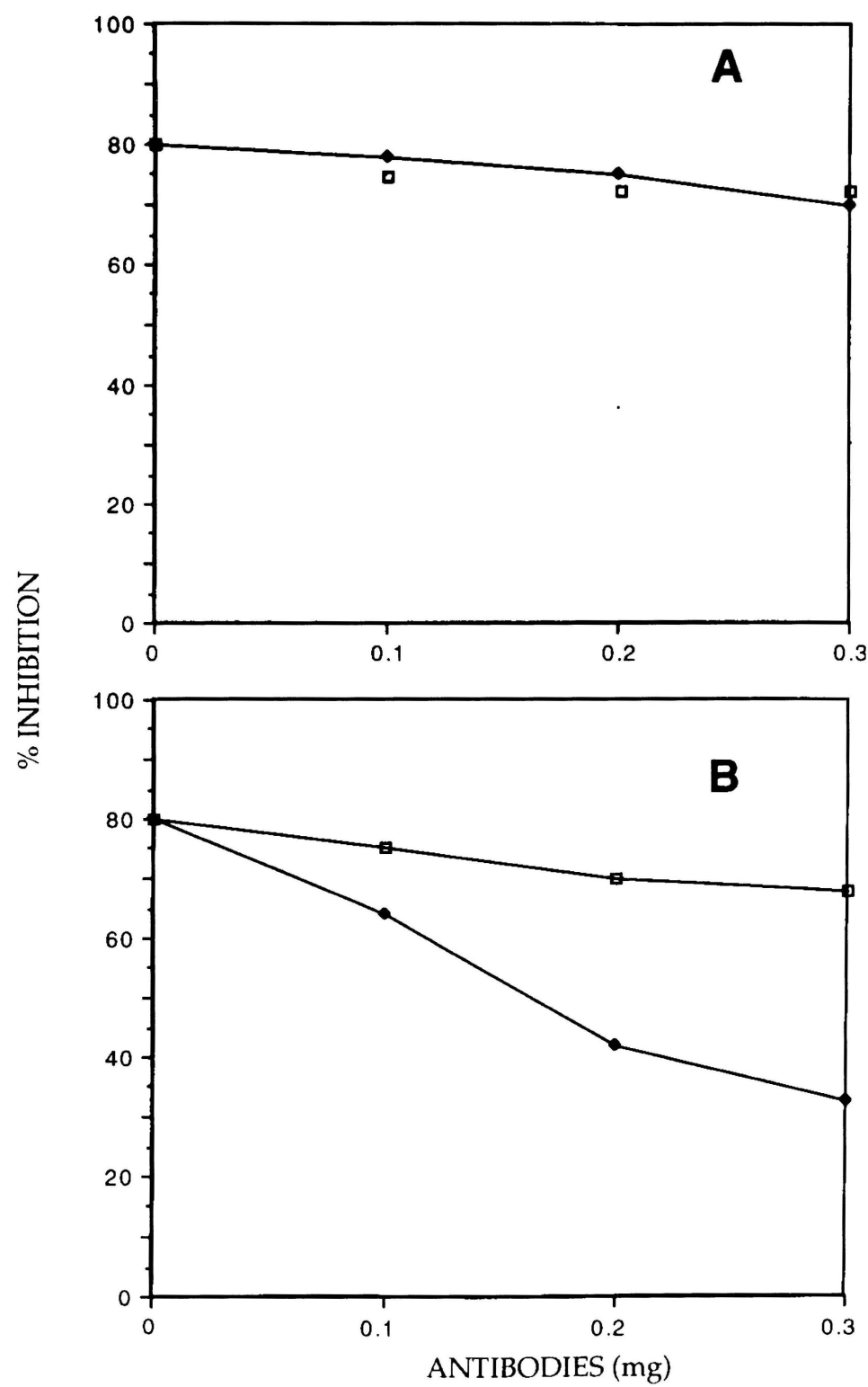

Figure 3. Effect of increasing quantities of anti-aprotinin antibodies $(\square)$ and anti-Inter- $\alpha$-Trypsin Inhibitor antibodies $\left(\right.$ ) on the antiproteolytic activity of the urinary trypsin inhibitors eluted from the RP-C ${ }_{18}$ column at a concentration of solvent B ranging between $30 \%$ and $40 \%$ (A) and between $40 \%$ and $80 \%$ (B). The effect was followed measuring the ability of the antibodies to remove the antitryptic activity of the inhibitors as previously reported (Fioretti et al., 1984; 1985).

the function of nervous system cells such as kallikrein and cathepsin-G (the UTI-like inhibitors). In addition, the urinary proteinase inhibitors are characterized by a relatively low molecular weight and appear potentially able to cross the blood-brain barrier. UTIlike inhibitors, deriving from proteolytic processing of the plasma protein Inter- $\alpha$ - 
Trypsin Inhibitor, are circulating molecules found in many biological fluids. In conclusion, the finding of increased levels of UTI-like inhibitors in urine of Alzheimer's disease patients seems to support the hypothesis that aberrant proteolytic processes could be involved in the pathogenesis of this neurological disorder. Nevertheless the results presented here are not sufficient for assigning a specific role to acid-stable proteinase inhibitors in degenerative phenomena of the nervous system, but can be seen as a starting point for further studies. In particular, it could be important to examine a larger sample of Alzheimer subjects and to perform a similar analysis on biological fluids (such as the cerebral spinal fluid) in a closer contact with the brain tissue.

\section{ACKNOWLEDGEMENTS}

The authors wish to thank Mr S Polzoni for his invaluable technical assistance. This work was supported, in part, by grants from the University of Camerino (E.F.) and from the Italian National Research Council (CNR) (S.B.).

\section{REFERENCES}

Abraham, R.C., Selkoe, D.J., Potter, H. (1988). Immunochemical identification of serine protease inhibitor $\alpha_{1}$-antichymotrypsin in the brain amyloid deposits of Alzheimer's disease. Cell, 52, 487-501.

Abraham, R.C., Potter, H. (1989). Alzheimer's disease: recent advances in understanding the brain amyloid deposits. Biotechnol., 7, 147-153.

American Psychiatric Association. (1980). Diagnostic and Statistical Manual of Mental Disorders. 3rd ed. Washington, DC, American Psychiatric Association.

Aoyagi, T., Wada, T., Nagai, M., KoJima, F., Harada, S., Takeuki, T., Takahashi, H., Hirokawa, T., Tsumita, T. (1990). Deficiency of kallikrein-like enzyme activities in cerebral tissue of patients with Alzheimer's disease. Experientia, 46, 94-97.

Chawla, R.K., Wadsworth, A.D., Rudman, D.J. (1978). Relation of the urinary cancer-related glycoprotein EDC 1 to plasma inter- $\alpha$-trypsin inhibitor. Immunol., 121, 1636-1639.

Eleuteri, A.M., Sparro, G., Angeletti, M., Fioretti, E. (1994). Kallikrein inhibitors in human urine: a possible regulation mechanism for the kallikrein-kinins system. Proceedings of the SIB-TUM meeting, Assisi (PG).

Fioretti, E., Citro, G., Ascoli, F. (1984). Immunochemical studies on the Kunitz-type inhibitors from bovine spleen. Prep. Biochem., 14, 389-401.

Fioretti, E., Angeletti, M., Citro, G., Ascoli, F. (1985). Characterization of three new Kunitz-type inhibitors from bovine spleen : preparation of specific antibodies. Prep. Biochem., 15, 211-219.

Fioretti, E., Eleuteri, A.M., Angeletti, M., Ascoli, F. (1993). High-performance liquid chromatographic separation of aprotinin-like inhibitors and their determination in very small amounts. J. Chrom. Biom. Appl., 617, 308-312.

Fritz, H., Wunderer, G. (1983). Biochemistry and applications of aprotinin, the kallikrein inhibitor from bovine organs. Drug Res., 33 (Suppl.), 479-494.

Gebhard, W., Hochstrasser, K. (1986). Inter- $\alpha$-trypsin inhibitor and its close relatives. In: Barrett, A.J., Salvesen, G. (Eds), Proteinase Inhibitors, Amsterdam. Elsevier, 389-401.

Huhtala, M.L., Pesonen, K., Kalkkinen, N., Stenman, U.H. (1982). Purification and characterization of a tumor-associated trypsin inhibitor from the urine of a patient with ovarian cancer. $J$. Biol. Chem., 257, 13713-13716.

Jochum, M., Witte, J., Duswald, K.H., Inthorn, D., Welter, H., Fritz, H. (1986). Pathobiochemistry of sepsis: role of proteinases, proteinase inhibitors and oxiding agents. Behring Inst. Mitt., 79, $121-130$. 
Kitaguchi, N., Takahashi, V., Tokushima, Y., Shiojiri, S., Ito, H. (1988). Novel precursor of Alzheimer's disease amyloid protein shows protease inhibitory activity. Nature, 331, 530-532.

Kizuki, K., Suzuki, T., Sugaya, K., Noguchi, T., Kudo, M. (1994). Tissue kallikrein in rat and mouse neurons. Brazilian J. Med. Biol. Res., 27, 1891-1896.

Krystosek, A., Seeds, N.W. (1984). Peripheral neurons and Schwann cells secrete plasminogen activator. J. Cell Biol., 98, 773-776.

Laemmli, U.K. (1970). Cleavage of structural proteins during the assembly of the head of bacteriophage T4. Nature, 227, 680-688.

Laskowski, J.M., Kato, I. (1980). Protein inhibitors of proteinases. Annu. Rev. Biochem., 49, $593-$ 626.

Masters, C.L., Simms, G., Weinmann, N.A., Multhaup, G., McDonald, B.L., Bayreuther, K. (1985). Amyloid plaque core protein in Alzheimer disease and Down syndrome. Proc. Natl. Acad. Sci. USA, 82, 4245-4249.

Matsuda, K., Ogawa, M., Murata, A., Kitahara, T., Kosaki, G. (1983). Elevation of serum immunoreactive pancreatic secretory trypsin inhibitor contents in various malignant diseases. Res. Commun. Chem. Pathol. Pharmacol.,40, 301-305.

McKhann, G., Drachman, D., Folstein, M., Katzman, R., Price, D., Stadlan, E.M. (1984). Clinical diagnosis of Alzheimer's disease report of the NINCS-ADRDA Work Group under the auspices of Department of Health and Human Service Task Force on Alzheimer's disease. Neurology, 34, 939-944.

Ogawa, M., Matsuura, N., Higashiyama, K., Mori, T. (1987). Expression of pancreatic secretory trypsin inhibitor in various cancer cells. Res. Commun. Chem. Pathol. Pharmacol., 55, 137140.

Okumichi, T., Nishiki, M., Takasugi, S., Toki, N., Ezaki, H. (1984). Isolation of urinary trypsin inhibitor-like inhibitor from human lung cancer tissue. Cancer Res., 44, 2011-2015.

Onitsuka, K., Sumi, H., Maruyama, M., Mihara, H. (1985). Increase of UTI activity in cases of malignant tumor. Jpn. J. Clin. Pathol., 33, 445-449.

Pittman, R.N. (1984). Neuron-target cell interactions may involve protease-inhibitor interactions. Soc. Neurosci. Abstr., 10, 662.

Pittman, R.N. (1985). Release of plasminogen activator and a calcium-dependent metalloprotease from cultured sympathetic and sensory neurons. Dev. Biol., 110, 91-101.

Pittman, R.N., Patterson, P.H. (1987). Characterization of an inhibitor of neuronal plasminogen activator released by heart cells. J. Neurosci., 7, 266-2673.

Ponte, P., Gonzalez-De Whitt, P., Schilling, J., Miller, J., Hsu, D., Greenberg, B., Davis, K., Wallace, W. (1988). A new A 4 amyloid mRNA contains a domain homologous to serine proteinase inhibitor. Nature, 331, 525-527.

Razzaboni, B.L., Papastoitsis, G., Koo, E., Abraham, C.R. (1992). A calcium-stimulated serine protease from monkey brain degrades the $\beta$-amyloid precursor protein. Brain Res., 589, $207-$ 216.

Shulman, N.R. (1955). A proteolytic inhibitor with anticoagulant activity separated from human urine and plasma. J. Biol. Chem., 213, 655-671.

Sumi, H., Maruyama, M., Tsushima, H. (1987). Studies on acid stable trypsin inhibitor in human urine, plasma, ascites and ovarian cancer. Biomed. Sci., 6, 117-120.

Tanzi, R.E., McClatchey, A.I., Lamperti, E.D., Villa-Komaroff, L., Gusella, J.F., Neve, R.L. (1988). Protease inhibitor domain encoded by an amyloid protein precursor mRNA associated with Alzheimer's disease. Nature, 331, 528-530.

Tomita, N., Doi, S., Higashiyama, M., Morimoto, H., Murotani, M., Kawasaki, Y., Shimano, T., Horii, A., Yokouchi, H., Ogawa, M., Mori, T., Matsubara, K. (1990). Expression of pancreatic secretory trypsin inhibitor gene in human colorectal tumor. Cancer, 66, 2144-2149.

Travis, J., Salvesen, G.S. (1983). Human plasma proteinase inhibitors. Annu. Rev. Biochem., 52, 655-709.

Yoshida, E., Sumi, H., Maruyama, M., Tsushima, H., Matsuoka, Y., Sugiki, M., Mihara, H. 
(1989). Distribution of acid stable trypsin inhibitor immunoreactivity in normal and malignant human tissues. Cancer, 64, 860-869. 


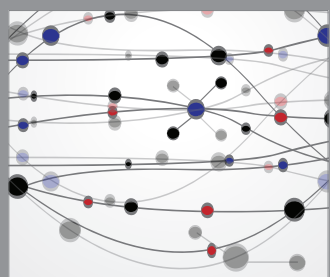

The Scientific World Journal
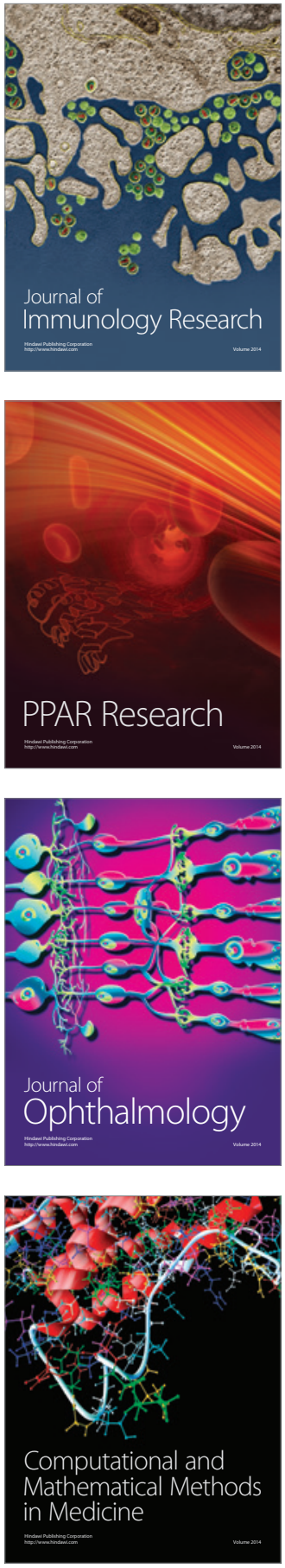

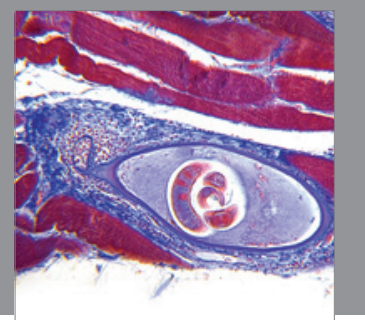

Gastroenterology

Research and Practice
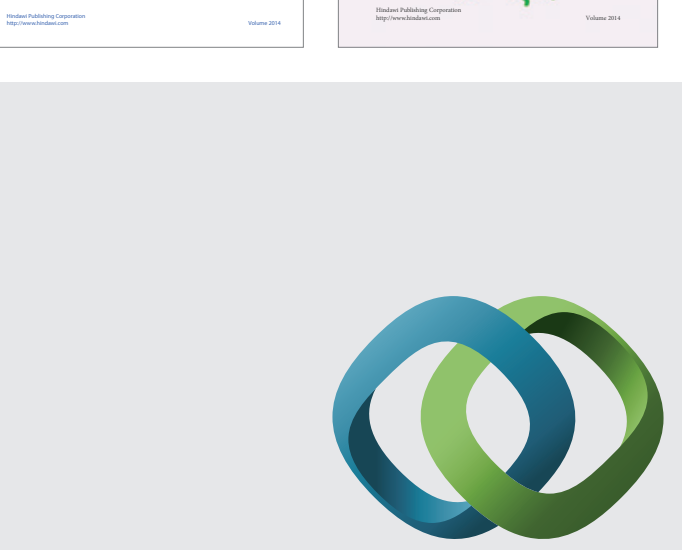

\section{Hindawi}

Submit your manuscripts at

http://www.hindawi.com
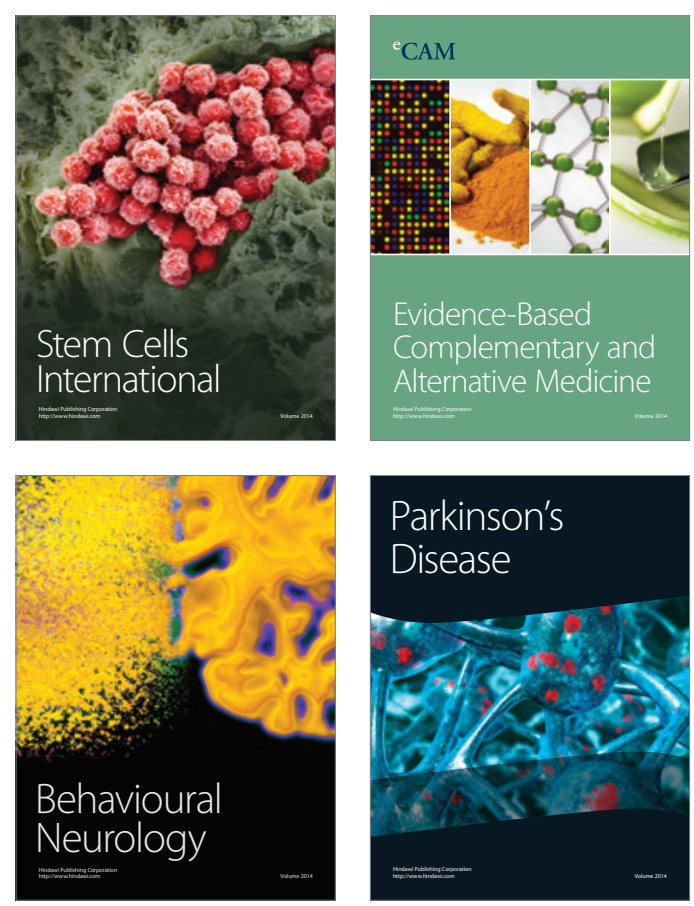

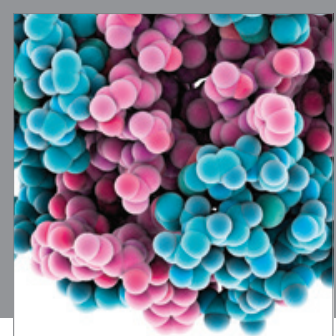

Journal of
Diabetes Research

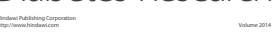

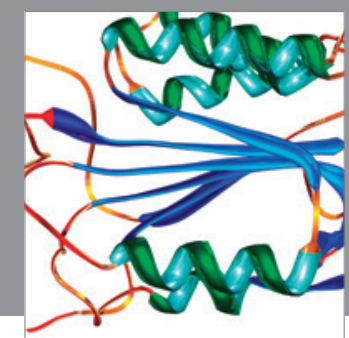

Disease Markers
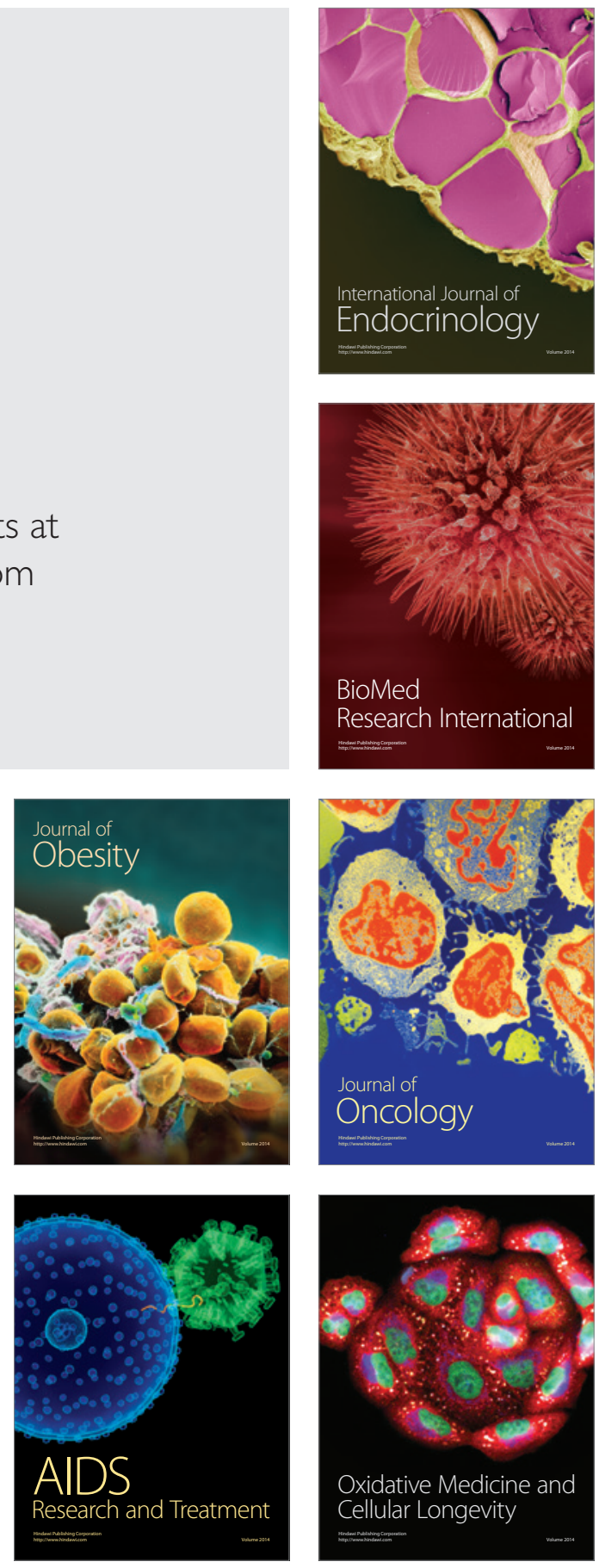